\title{
Evaluation of the Lower Urinary Tract before Renal Transplantation: To Which Patients? How?
}

\author{
Adnan Simsir ${ }^{1 *}$, Hamad Dheir ${ }^{2}$, Rashad Mammadov ${ }^{1}$, Cuneyt Hoscoskun ${ }^{3}$, Huseyin Toz ${ }^{2}$, \\ Ercan Ok ${ }^{2}$, Ceyhun Ozyurt ${ }^{1}$ \\ ${ }^{1}$ Department of Urology, Ege University School of Medicine, Izmir, Turkey \\ ${ }^{2}$ Division of Nephrology, Ege University School of Medicine, Izmir, Turkey \\ ${ }^{3}$ Department of General Surgery, Ege University School of Medicine, Izmir, Turkey \\ Email: *adnan.simsir@ege.edu.tr
}

Received June 3, 2012; revised July 5, 2012; accepted July 13, 2012

\begin{abstract}
Purpose: The evaluation of lower urinary tract (LUT) before transplantation is not required, except in patients with a history of LUT disfunction (LUTD). In this study, we adressed the necessity for lower urinary system evaluation in transplantation candidates classified according to their etiology. Materials and Methods: Sixty-two patients were prospectively evaluated. The patients were evaluated at 2 months preoperatively and at 6 months post transplantation using questionnaires, bladder diary and urodynamic studies. The end stage renal disease (ESRD) etiologies of the patients were divided into three groups as follows: patients with a parenchymal disease, patients with LUTD and those with unknown etiology and the obtained results were evaluated accordingly. Results: The evaluation of 50 patients revealed significant differences in ICIQ-SF $(p<0.001)$, IPSS $(p<0.001)$, daily urine volumes $(p=0.05)$, maximum urinary flow rate $(p=0.03)$, PVR, the number of patients with VUR and all cystometric parameters between the patient groups divided according to the etiology. The comparison of the groups to each other revealed no statistically significant differences in all parameters between the LUTD group and the patient groups with unknown etiology. Considering that the results from questionnaires of patients with a single voided volume of $100 \mathrm{cc}$ or greater on the bladder diary would be scientifically more significant, they were evaluated separately. Thus, even though the results from questionnaires did not differ between the group with unknown etiology and the LUTD group, there were significant differences when compared to patients who developed ESRD due to a parenchymal disease. Similar findings were found between the results of urodynamic study. Conclusions: The evaluation of lower urinary tract in patients with a history of LUTD and in those with unknown etiology should be initially evaluated by noninvasive methods such as questionnaires and uroflowmetry.
\end{abstract}

Keywords: Renal Transplantation; Lower Urinary Tract; Evaluation; Urodynamics

\section{Introduction}

Renal transplantation, which is the gold standard treatment of end stage renal dysfunction (ESRD), has today become a routine operation due to newly developed immunosuppressive agents and antibiotics, improved quality of intensive care follow-up, advances in surgical techniques and increased experience [1,2]. Since lower urinary tract dysfunction (LUTD) is not involved in absolute or relative contraindications for transplantation, preoperative evaluation of the lower urinary tract (LUT) is not performed routinely in most centers [3]. Several authorities, however, suggest that patients with a medical history of urological disease, pyuria, massive crystalluria and those with a more than 6-month history of anuria should

*Corresponding author. be evaluated by voiding-cystourethrogram (VCUG) [4-6]. An evaluation by VCUG aims to preoperatively identify patients with LUT problems and to take necessary precautions to prevent graft damage and loss after transplantation. In this study, we investigated in which patients and by which technique pretransplantation evaluation of the LUT should be performed and investigated whether there was a change in the functioning of the lower urinary tract after transplantation, and thus the reliability of preoperative values.

\section{Materials and Methods}

Sixty-two patients who were planned to undergo renal transplantation with the diagnosis of ESRD in a single center between December 2007-November 2009 were enrolled in this study. The patients were examined pro- 
spectively at 2 months preoperatively and at 6 months post transplantation. Two patients who had early rejection were excluded from the study, which eventually included a total of 60 patients. Table 1 summarizes the characteristics of the patients.

The patients were first seen 2 months before the scheduled transplantation date. During this first evaluation, medical history of the patients were recorded and urine culture antibiograms were taken. Patients with a significant colony count $\left(>10^{5}\right)$ on culture antibiogram received antibiotherapy. After confirming that their urine was sterile, ICIQ-SF (incontinence questionnaire short form), 3-day voiding diary and IPSS (international prostate symptom score index) assessing lower urinary tract symptoms were given. Based on the data from the bladder diary, 50 patients with a single voided volume of 100 or greater underwent uroflowmetry, post-void residual volume (PVR) measurement and filling and voiding cystometry (voiding cystography was simultaneously performed by video-urodynamics), respectively. An informative uroflowmetry needs a voided volume of greater than $100 \mathrm{cc}$, which was therefore taken as the cut-off value [7]. A MMS ${ }^{\mathrm{R}}$ (Medical Measurement Systems) urodynamic device was used for urodynamic studies and Bladder Scan was used for post-void residual urine measurement.

Table 1. Patients caracteristics.

\begin{tabular}{cc}
\hline Caracteristics & $\mathrm{n}(\%)$ \\
\hline Number of patients & 60 \\
Female & $24(40)$ \\
Male & $36(60)$ \\
Age (mean) & $34.45(18-70)$ \\
Period of dialysis (month) & $29.5(0-216)$ \\
Procedure of dialysis & $50(83.3)$ \\
Hemodialysis & $10(16.7)$ \\
Periton dialysis & $24(40)$ \\
Etiology of ESRD & \\
Parenchymal diseases & $15(25)$ \\
LUTD & $21(35)$ \\
Unknown etiology & $6.48(4.78-8.4)$ \\
Mean creatinine level (mg/dl) & \\
Urine level (cc/day) $^{* * *}$ & $4(6.7)$ \\
$<100$ & $26(43.3)$ \\
$100-500$ & $22(36.7)$ \\
$500-1000$ & $8(13.3)$ \\
$>1000$ & $10(16.7)$ \\
Single voided volume (cc) $_{>100}^{100}$ & $50(83.3)$ \\
\hline
\end{tabular}

\footnotetext{
*: End stage renal disease; ${ }^{* *}:$ Lower urinary tract disfunction (include 12 patients with neurogenic bladder and VUR \& 3 patients with posterior uretral valve); ${ }^{* * *}$ : Mean value of three days, according to bladder diary.
}

To allow sound scientific interpretation, these 50 patients were divided into 3 groups according to their etiology. Accordingly, patients who were diagnosed with a parenchymal disease and thus be candidates for transplantation constituted Group 1, those who developed renal failure due to LUTD (valve, neurogenic bladder and/or vesicoureteral reflux (VUR)) and thus be candidates for transplantation constituted Group 2 and transplantation candidates with unknown etiology constituted Group 3. The obtained findings were investigated in order to evaluate the LUTs of these 50 patients with a mean voided volume of $100 \mathrm{cc}$ who were divided into groups according to their etiology. In addition, all patients (including anuric patients) were invited for a follow-up assessment six months after transplantation and all above mentioned procedures were performed again and the results obtained were evaluated.

SPSS version 15.0 was used for data analysis and a $\mathrm{p}$ value of $\leq 0.05$ was considered statistically significant. Student's t-test, Mann-Whitney U test, ANOVA and Fisher's exact test were the methods of choice.

\section{Results}

The mean age of patients in this study was 34.45 (range 18 - 70). Blood creatinine levels, which were $6.48 \mathrm{mg} / \mathrm{dl}$ (4.78 - 8.4) before transplantation, were measured to be $1.2 \mathrm{mg} / \mathrm{dl}(0.82$ - 2.21) after transplantation.

The evaluation of 50 patients revealed significant differences in ICIQ-SF $(p<0.001)$, IPSS $(p<0.001)$, daily urine volumes $(\mathrm{p}=0.05)$, maximum urinary flow rate $(\mathrm{p}$ $=0.03$ ), PVR, the number of patients with VUR and all cystometric parameters between the patient groups divided according to the etiology. Interestingly, the comparison of the groups to each other revealed no statistically significant differences in all parameters between the LUTD group (Group 2) and the patient groups with unknown etiology (Group 3) (Table 2). Cystographic examination revealed that the groups did not differ in cystographic bladder capacity, however, it is noteworthy that the number of VUR in Group 1 was significantly lower than that in the other groups.

Additionally, the comparison of VCUG findings (cystometric bladder capacity and the number of patients with VUR) and noninvasive methods, which was one of the parameters tested, in these 50 patients showed a significant correlation with ICIQ-SF score, IPSS, mean voided volume, maximum flow rate and post-void residual urine volume values.

Furthermore, re-evaluation of all patients at 6 months post transplantation and the comparison between the parameters revealed significant improvement in flow rate and bladder compliance in Group $1(\mathrm{p}<0.001, \mathrm{p}=0.05)$, whereas statistically significant changes were found in all 
Table 2. The changes in pretransplantation values of the parameters investigated according to the etiology ${ }^{*}$.

\begin{tabular}{|c|c|c|c|c|}
\hline Parametreler & $\begin{array}{c}\text { Parenchymal } \\
\text { etiology }\end{array}$ & LUTD & $\begin{array}{l}\text { Unknown } \\
\text { etiology }\end{array}$ & \\
\hline $\begin{array}{l}\text { Number of } \\
\text { patients }\end{array}$ & 19 & 13 & 18 & \\
\hline ICIQ SF (mean) & 2.2 & 15.2 & 10.4 & $<0.001$ \\
\hline IPSS (mean) & 4.6 & 19.3 & 13.1 & $<0.001$ \\
\hline \multicolumn{5}{|l|}{ Bladder diary } \\
\hline $\begin{array}{l}\text { Voided volume } \\
\qquad(\mathrm{cc})\end{array}$ & 104 & 175 & 235 & 0.046 \\
\hline $\begin{array}{l}\text { Number of } \\
\text { void (day) }\end{array}$ & 0.7 & 0.9 & 1.1 & 0.43 \\
\hline $\begin{array}{l}\text { Urine level } \\
\text { (cc/day) }\end{array}$ & 265 & 370 & 402 & 0.04 \\
\hline $\begin{array}{l}\text { Max. flow } \\
\text { rate }(\mathrm{ml} / \mathrm{s})\end{array}$ & 12.3 & 4.2 & 6.2 & 0.039 \\
\hline $\mathrm{PVR}^{* *}(\mathrm{cc})$ & 70 & 278 & 175 & $<0.001$ \\
\hline $\begin{array}{l}\text { Cystographic } \\
\text { capacity (cc) }\end{array}$ & 315 & 203 & 280 & 0.09 \\
\hline $\begin{array}{l}\text { Number of } \\
\text { VUR (\%) }\end{array}$ & $1(5.6)$ & 11 (91.6) & $11(78.6)$ & $<0.001$ \\
\hline \multicolumn{5}{|l|}{ Cystometry } \\
\hline Max capacity & 301 & 197 & 211 & $<0.001$ \\
\hline Max p detrusor & 17.4 & 31.1 & 28.5 & $<0.001$ \\
\hline Compliance & 10.2 & 6.4 & 6.9 & 0.05 \\
\hline $\mathrm{BCI}^{* * *}$ & 110 & 183 & 161 & 0.040 \\
\hline
\end{tabular}

*: Investigated 50 patients (single voided urine level $>100 \mathrm{cc}$ ); ${ }^{* *}:$ Post voiding residue urine; ${ }^{* * *}$ : Bladder contractibilty index. parameters except ICIQ scores and BCI in Group 2 and in all parameters in Group 3 (Table 3).

\section{Discussion}

Pretransplantation evaluation of patients is a process that includes long, invasive and expensive procedures [8-11]. Patients with a history of urological disease, pyuria, massive crystalluria and those with a more than 6-month history of anuria are evaluated by physical examination, urine culture, urine analysis, renal ultrasound and VCUG [5-7]. However, should only these patients be evaluated? And, can other non-invasive methods providing objective information be used instead of VCUG which provides objectively the most important data compared to the above mentioned methods?

In this study aiming to address these questions, we concluded that lower urinary tract evaluation is not required in patients who developed ESRD due to a parenchymal disease. The literature review revealed that almost all authors held similar views to those of us [12-15]. However, it is of interest to note in the present study that most findings obtained from the evaluation of transplantation candidates with unknown etiology were similar to those of candidates with LUTD etiology. Thus, the evaluation of the LUT should absolutely be performed in this group of patients. But how? Should it be performed by VCUG which is actually an invasive method? It is a well-recognized fact that VCUG causes severe infections in patients whose defense mechanisms against infection in the urinary tract have been compromised by chronic renal failure. This study showed a significant correlation between VCUG findings and IPSS, ICIQ-SF, daily voided volume from the bladder diary, maximum flow rate and

Table 3. Posttransplantation changes in the parameters investigated.

\begin{tabular}{|c|c|c|c|c|c|c|c|c|c|}
\hline \multirow[t]{2}{*}{ Parameters } & \multicolumn{3}{|c|}{ Parenchymal etiology } & \multicolumn{3}{|c|}{ LUTD } & \multicolumn{3}{|c|}{ Unknown etiology } \\
\hline & Pre Tx & Post Tx & $\mathrm{p}$ & Pre Tx & Post Tx & $\mathrm{p}$ & Pre Tx & Post Tx & $\mathrm{p}$ \\
\hline IPSS & 4.6 & 3.1 & 0.46 & 19.3 & 15.4 & $<0.001$ & 13.1 & 5.2 & 0.05 \\
\hline Max. flow rate & 12.3 & 18.1 & $<0.001$ & 4.2 & 15.4 & $<0.001$ & 6.2 & 17.1 & $<0.001$ \\
\hline PVR & 70 & 63 & 0.87 & 278 & 82 & $<0.001$ & 175 & 38 & $<0.001$ \\
\hline $\begin{array}{c}\text { Cystographic } \\
\text { capacity }\end{array}$ & 315 & 410 & 0.14 & 203 & 320 & 0.038 & 280 & 397 & 0.04 \\
\hline \multicolumn{10}{|l|}{$\begin{array}{c}\text { Urodynamic } \\
\text { values }\end{array}$} \\
\hline Max. capacity & 301 & 365 & 0.32 & 197 & 304 & $<0.001$ & 211 & 362 & $<0.001$ \\
\hline Max. p detrusor & 17.4 & 14.1 & 0.73 & 31.1 & 17.8 & 0.04 & 28.5 & 13.2 & $<0.001$ \\
\hline Compliance & 10.2 & 14.1 & 0.05 & 6.2 & 13.1 & $<0.001$ & 6.4 & 15.0 & $<0.001$ \\
\hline $\mathrm{BCI}$ & 110 & 105 & 0.91 & 183 & 142 & 0.07 & 161 & 127 & 0.05 \\
\hline
\end{tabular}


PVR levels. Given that all these parameters are noninvasive, even though questionnaires are not defined as an alternative to VCUG, it is not recommended to perform VCUG in the absence of abnormal findings in these parameters. A study by Yang et al. reported that the most common abnormality found on VCUG before transplantation was decreased bladder capacity [15]. Moreover, VUR was found to be the second most common abnormal finding on VCUG. Among the parameters investigated in this study, uroflowmetry, bladder diary and functional bladder capacity can be detected non-invasively (in patients with a voided volume of $100 \mathrm{cc}$ or greater). In patients with elevated PVR by Bladder Scan in whom bladder dysfunction is suspected, much more reliable data can be obtained by using urodynamic techniques (videourodynamics) instead of VCUG. In addition, VUR, which is another parameter detected by a VCUG, if isolated (if not accompanied by bladder dysfunction), is not of clinical importance since uretero-vesical anastomosis is already performed during transplantation, which makes us consider that the necessity of VCUG should be questioned.

Another important result obtained in the second stage of this study was increased urine volume as well as a significant improvement in almost all parameters including cystometric findings at 6 months post transplantation. This result suggests that surgeons can be more aggressive in planning transplantation in patients with decreased bladder capacity and compliance.

\section{Conclusion}

During the evaluation of patients prior to renal transplanttation, lower urinary tract evaluation is not required in patients with no complaints related with the lower urinary tract and with no history of lower urinary tract dysfunction who developed ESRD due to a parenchymal disease. Particularly, patients with a voided volume of $100 \mathrm{cc}$ or greater, those with a history of LUTD and those with unknown etiology are recommended to be initially evaluated with questionnaires, uroflowmetry and PVR. In this situation, in the presence of data indicating LUTD, videourodynamic examinations, instead of VCUG, should be performed to completely identify the problem.

\section{REFERENCES}

[1] R. Shapiro, "Outcome after Renal Transplantation," In: R. Shapiro, R. L. Simmons and T. E. Starzl, Eds., Renal Transplantation, Appleton Lange, London, 1997, p. 1.

[2] B. L. Kasiske, "The Evaluation of Prospective Renal Transplant Recipients and Living Donors," Surgical Clinics of North America, Vol. 78, No. 1, 1998, pp. 27-39. doi:10.1016/S0039-6109(05)70632-0
[3] M. Campise, "Selection and Preparation of the Recipient," In: B. D. Kahan and C. Ponticelli, Eds., Principles and Practise of Renal Transplantation, Martin Dunitz, London, 2001, pp. 89-143.

[4] J. McCauley, "Evaluation of Potential Renal Allograft Recipient," In: R. Shapiro, R. L. Simmons and T. E. Starzl, Eds., Renal Transplantation, Appleton Lange, London, 1997, pp. 43-72.

[5] R. E. Power, D. P. Hickey and D. M. Little, "Urological Evaluation Prior to Renal Transplantation," Transplantation Proceedings, Vol. 36, No. 10, 2004, pp. 2962-2967. doi:10.1016/i.transproceed.2004.11.006

[6] K. Shandera, A. Sago, J. Angs, et al., "An Assesment of the Need for Voiding Cystourethrogram for Urologic Screening, Prior to Renal Transplantation," Clinical Transplantation, Vol. 7, No. 4, 1993, pp. 299-301.

[7] W. Schafer, P. Abrams, L. Liao, et al., "Good Urodynamic Practices: Uroflowmetry, Filling Cystometry, and Pressure-Flow Studies," Neurourology and Urodynamics, Vol. 21, No. 3, 2002, pp. 261-274.

doi:10.1002/nau.10066

[8] K. Shandera, T. A. Rozanski and G. Jaffers, "The Necessity of Voiding Cystourethrogram in the Pretransplant Urologic Evaluation," Urology, Vol. 47, No. 2, 1996, pp. 198-200. doi:10.1016/S0090-4295(99)80415-4

[9] A. Sharfuddin, "Imaging Evaluation of Kidney Transplant Recipients," Seminars in Nephrology, Vol. 31, No. 3, 2011, pp. 259-271. doi:10.1016/j.semnephrol.2011.05.005

[10] B. Kalb, D. R. Martin, K. Salman, P. Sharma, J. Votaw and C. Larsen, "Kidney transplantation: Structural and Functional Evaluation Using MR Nephro-Urography," Journal of Magnetic Resonance Imaging, Vol. 28, No. 4, 2008, pp. 805-822. doi:10.1002/jmri.21562

[11] S. M. Friedewald, E. P. Molmenti, J. J. Friedewald, M. R. Dejong and U. M. Hamper, "Vascular and Nonvascular Complications of Renal Transplants: Sonographic Evaluation and Correlation with Other Imaging Modalities, Surgery, and Pathology," Journal of Clinical Ultrasound, Vol. 33, No. 3, 2005, pp. 127-139. doi:10.1002/jcu.20105

[12] H. P. Koo, T. E. Bunchman, J. T. Flynn, et al., "Renal Transplantation in Children with Severe Lower Urinary Trcat Disfunction," The Journal of Urology, Vol. 161, No. 1,1999 , pp. $240-245$. doi:10.1016/S0022-5347(01)62119-0

[13] J. Barry, "Kidney Transplantation into Patients with Abnormal Bladders," Transplantation, Vol. 77, No. 3, 2004, pp. 1120-1123. doi:10.1097/01.TP.0000116711.59454.F1

[14] R. H. Jefferson and J. R. Burns, "Urologic Evaluation of Adult Renal Transplant Recipients," The Journal of Urology, Vol. 153, No. 3, 1995, pp. 615-618. doi:10.1097/00005392-199503000-00016

[15] C. C. Yang, M. C. Rohr and D. G. Assimos, "Pretransplant Urologic Evaluation," Urology, Vol. 43, No. 2, 1994, pp. 169-173. doi:10.1016/0090-4295(94)90038-8 